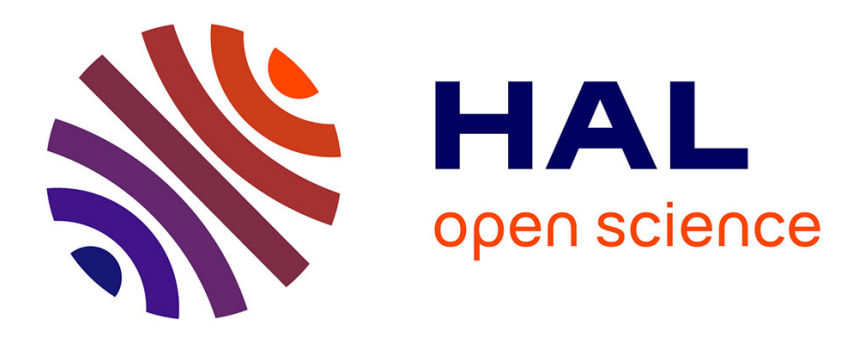

\title{
Variété des modes d'organisation des premiers cycles et réussite universitaire
}

Jean-Pierre Jarousse, Christophe Michaut

\section{To cite this version:}

Jean-Pierre Jarousse, Christophe Michaut. Variété des modes d'organisation des premiers cycles et réussite universitaire. Revue Française de Pédagogie, 2001. halshs-01225538

\section{HAL Id: halshs-01225538 \\ https://shs.hal.science/halshs-01225538}

Submitted on 6 Nov 2015

HAL is a multi-disciplinary open access archive for the deposit and dissemination of scientific research documents, whether they are published or not. The documents may come from teaching and research institutions in France or abroad, or from public or private research centers.
L'archive ouverte pluridisciplinaire HAL, est destinée au dépôt et à la diffusion de documents scientifiques de niveau recherche, publiés ou non, émanant des établissements d'enseignement et de recherche français ou étrangers, des laboratoires publics ou privés. 


\title{
Variété des modes d'organisation des premiers cycles et réussite universitaire
}

\author{
Jean-Pierre Jarousse \\ Christophe Michaut
}

Depuis 1968, les réformes concernant l'enseignement supérieur ont accordé une autonomie croissante aux universités en matière d'organisation pédagogique. La question qui se pose aujourd'hui est celle de savoir comment varie l'organisation du DEUG d'une filière à l'autre et surtout d'un établissement à l'autre pour de mêmes filières. Cet article, basé sur l'exploitation d'un questionnaire adressé aux responsables de premier cycle des universités françaises, montre qu'il existe aujourd'hui, sur des points importants de l'organisation des DEUG (organisation générale, administration, structure et contenu des enseignements, certification, dispositifs d'aides aux étudiants), des différences importantes entre établissements. Si ce premier résultat met à mal l'image traditionnelle des "diplômes nationaux", il en est de même de l'absence de relation statistique entre les variétés en matière d'organisation et les variétés constatées en matière de réussite. Ce dernier résultat est éventuellement imputable à l'imperfection des données mais il peut tout autant suggérer que, au-delà des conditions de la réussite, les modalités de la réussite (contenu des examens, niveau d'exigence, barèmes,...) varient elles-mêmes d'un établissement à l'autre.

Mots-clés : curricula, DEUG, organisation pédagogique, réussite, supérieur premier cycle.

\section{INTRODUCTION}

De la réforme Fouchet (1966) à la réforme Bayrou (1997), en passant successivement par la loi d'orientation de 1968 ( $E$. Faure), la réforme de 1973, la loi d'orientation de 1984 (A. Savary) et la réforme Jospin-Lang de 1992, les universités ont progressivement acquis une réelle autonomie financière et surtout pédagogique. Depuis 1968, l'organisation des diplômes ne relève plus a priori des instances nationales mais de chaque établissement universitaire chargé de délivrer des diplômes nationaux habilités a posteriori par le ministère. La réforme de 1973 fixe un cadre à cette autonomie pédagogique en précisant, pour chaque filière, la durée d'enseignement minimale ainsi que la liste des disciplines fondamentales et optionnelles tout en laissant aux établissements le soin de définir eux-mêmes le volume horaire et l'importance certificative de chaque matière enseignée. La loi d'orientation de 1984 renforce 
cette autonomie pédagogique en permettant aux établissements de choisir une organisation générale du DEUG basée sur deux années ou quatre semestres. Avec l'instauration des modules, qui regroupent "des enseignements comportant entre eux une cohérence scientifique et pédagogique" (art. 4, arrêté du 26 mai 1992), la réforme JospinLang va encore plus loin dans cette direction en confiant aux établissements la définition des modules et de leurs modalités d'obtention. La réforme Bayrou, la plus récente, ne revient pas sur cette liberté accordée aux universités même si elle précise davantage les objectifs pédagogiques assignés aux regroupements de disciplines (les unités d'enseignement qui remplacent les modules) et leur succession dans l'année universitaire (semestrialisation).

Comment les universités ont-elles utilisé ces libertés en matière d'organisation des diplômes et des examens? Existe-t-il aujourd'hui des variations importantes quant à l'organisation du DEUG d'une filière à l'autre, et surtout d'un établissement à l'autre pour de mêmes filières ? Ces variétés peuvent-elles rendre compte, au moins partiellement, des écarts de réussite observés selon les sites ? Cet article présente les résultats d'une recherche visant à répondre à ces différentes questions. L'analyse repose sur l'exploitation d'un questionnaire adressé à tous les responsables de DEUG des universités françaises. La relative faiblesse du taux de réponse ne permettra malheureusement pas de dresser un panorama exhaustif des universités ni, bien sûr, d'apporter des réponses définitives aux questions posées précédemment. Le nombre de questionnaires dispo- nibles (155) est cependant tout à fait suffisant pour que les résultats présentés donnent des indications pertinentes sur la situation actuelle.

L'article est divisé en deux parties : la première fait le point sur les variétés constatées en matière d'organisation pédagogique des DEUG par filière et par établissement (organisation générale, administration, modalités de certification, dispositifs d'aide à la réussite) tandis que la seconde partie examine les relations entre organisation et réussite.

\section{L'HÉTÉROGÉNÉITE DU FONCTIONNEMENT PÉDAGOGIQUE DES DEUG}

Le fonctionnement pédagogique du DEUG est étudié ici sous quatre dimensions: l'organisation générale du cycle, les curricula, les modes de certification et les dispositifs d'aides à la réussite.

\section{L'organisation générale du DEUG}

Constitué de deux années (ou de deux niveaux, voire de quatre périodes suivant l'appellation adoptée par chaque filière), le DEUG débute le plus souvent par une phase d'accueil des étudiants durant laquelle sont présentés assez systématiquement le fonctionnement général de la première année (déroulement, emploi du temps, organisation des filières, ressources documentaires, dispositifs d'aide méthodologique, etc.) et les modalités du contrôle des connaissances. Les perspectives universitaires $(55,3 \%)$, et plus rare-

Tableau I. - Volume annuel d'enseignement (en heures) (1)

\begin{tabular}{|l|c|c|c|c|c|c|c|}
\hline \multicolumn{1}{|c|}{$\mathrm{N}=147$} & AES & Psychologie & $\begin{array}{c}\text { Sciences } \\
\text { de la Vie } \\
\text { Sciences } \\
\text { de la Terre }\end{array}$ & $\begin{array}{c}\text { Autres } \\
\text { DEUG } \\
\text { scientifiques }\end{array}$ & $\begin{array}{c}\text { Lettres } \\
\text { Langues- } \\
\text { Arts }\end{array}$ & $\begin{array}{c}\text { Droit } \\
\text { Économie } \\
\text { et Gestion }\end{array}$ & $\begin{array}{c}\text { Autres } \\
\text { DEUG } \\
\text { SHS }\end{array}$ \\
\hline Moyenne & 501,2 & 384,2 & 558,1 & 579,2 & 450,9 & 530,5 & 398,9 \\
\hline Écart type & 49,6 & 39,7 & 39,2 & 24,2 & 63,2 & 71,3 & 34,7 \\
\hline Minimum & 437 & 304 & 479 & 525 & 299 & 410 & 322 \\
\hline Maximum & 626 & 449 & 657 & 617 & 625 & 656 & 475 \\
\hline
\end{tabular}

(1) Certains regroupements ont été nécessaires en raison du nombre réduit d'observations : Autres DEUG Scientifiques (10) : STAPS (6), TI (2), MIAS (2) et MASS (2) ; Lettres-Langues-Arts (28) : Lettres modernes et classiques (9), LEA (10), LLCE (7) et Arts (2); Droit et Économie (11); Autres DEUG SHS (26): Histoire (11), Géographie (14) et SHS pluridisciplinaire (1). 
Tableau II. - Proportion de Cours Magistraux selon les filières

\begin{tabular}{|l|c|c|c|c|c|c|c|}
\hline \multicolumn{1}{|c|}{$\mathrm{N}=141$} & AES & Psychologie & $\begin{array}{c}\text { Sciences } \\
\text { de la Vie } \\
\text { Sciences } \\
\text { de la Terre }\end{array}$ & $\begin{array}{c}\text { Autres } \\
\text { DEUG } \\
\text { scientifiques }\end{array}$ & $\begin{array}{c}\text { Lettres } \\
\text { Langues- } \\
\text { Arts }\end{array}$ & $\begin{array}{c}\text { Droit Economie } \\
\text { et Gestion }\end{array}$ & $\begin{array}{c}\text { Autres } \\
\text { DEUG } \\
\text { SHS }\end{array}$ \\
\hline Moyenne (en \%) & 65,6 & 58,8 & 45,8 & 42,0 & 37,8 & 71,0 & 52,0 \\
\hline Écart type & 7,6 & 15,0 & 6,7 & 11,4 & 16 & 7,4 & 14,1 \\
\hline
\end{tabular}

ment les perspectives professionnelles (31,6\%), sont également abordées lors de cette réunion d'accueil. Les étudiants suivent en moyenne 25,4 semaines d'enseignement entrecoupées de 3,2 semaines d'examens et d'une semaine et demie consacrée aux révisions. Les variations du nombre de semaines d'enseignement entre les filières et entre universités pour les mêmes filières sont relativement faibles. En revanche, le volume annuel d'enseignement varie fortement entre les filières mais également, à filière identique, entre les universités. C'est dans les disciplines scientifiques que la durée d'enseignement est la plus importante et la plus homogène. Dans les filières littéraires, juridiques ou économiques, le volume horaire annuel varie fortement d'une université à l'autre avec des écarts parfois spectaculaires entre les situations extrêmes.

S'agissant du type de cours, certains départements universitaires privilégient les cours magistraux alors que d'autres offrent davantage de travaux dirigés. Par le passé les filières littéraires (Lettres modernes, Lettres classiques) délivraient principalement un enseignement magistral. La situation est différente aujourd'hui avec cependant une forte variété d'une université à l'autre. Cette variété dans le type d'enseignement s'observe également au sein des filières scientifiques (en particulier les " autres DEUG scientifiques "). Les filières de Droit, Économie et AES se distinguent des autres par un recours plus important au cours magistral et par une plus grande homogénéité inter-sites. Les filières de Sciences Humaines sont sur ce plan dans une situation intermédiaire : la part des cours magistraux y est en moyenne élevée mais avec une forte dispersion d'une université à l'autre: en Psychologie, par exemple, la part des CM dans le volume total d'enseignement peut varier de 33 à $85 \%$.

Les filières à forts effectifs n'adoptent pas un système privilégiant les CM. De même, les antennes universitaires ne comptent pas proportionnellement plus de TD dans le volume total des cours. L'encadrement des étudiants dépend évidemment de la nature des enseignements. Sur l'échantillon étudié le nombre moyen d'étudiants par groupe de TD est de 36,5 (29,7 en référence aux seuls étudiants présents aux examens).

Une autre dimension de l'encadrement des étudiants de première année concerne le statut des enseignants et notamment la proportion de Maîtres de Conférences et de Professeurs dans l'ensemble des enseignants. Là encore des disparités apparaissent puisque ces enseignants sont plus nombreux à enseigner en première année de Sciences de la Vie (en moyenne $64 \%$ ) qu'en Lettres (46\%) ou qu'en Droit et AES (34,2\%). Si les écarts inter-universités sont prononcés, ils ne dépendent pas de la taille de l'université. Sur ce plan, les antennes ne se différencient pas des universités " mères".

\section{La diversité des curricula}

Trois caractéristiques des curricula peuvent être distinguées: le type d'enseignement (cours communs/options), le degré de spécialisation des filières (orientation en fin de premier semestre) et le statut des disciplines (majeures/mineures).

La proportion d'enseignements optionnels dans le volume total des enseignements est relativement élevée en Sciences Humaines. À l'inverse, en AES ou en Droit, l'enseignement est avant tout un enseignement commun à tous les étudiants. Mais ceci est extrêmement variable suivant les universités. En AES par exemple, 11 universités sur 25 n'offrent pas d'enseignements optionnels alors que dans 8 universités ils représentent plus de $10 \%$ du volume total d'enseignement. L'offre de formation optionnelle a tendance à être liée à la taille de l'université. II est bien sûr plus aisé de 
Tableau III. - Part (\%) du volume des enseignements optionnels dans le volume d'enseignement annuel

\begin{tabular}{|l|c|c|c|c|c|c|c|}
\hline \multicolumn{1}{|c|}{$\mathrm{N}=140$} & AES & Psychologie & $\begin{array}{c}\text { Sciences } \\
\text { de la Vie } \\
\text { Sciences } \\
\text { de la Terre }\end{array}$ & $\begin{array}{c}\text { Autres } \\
\text { DEUG } \\
\text { scientifiques }\end{array}$ & $\begin{array}{c}\text { Lettres } \\
\text { Langues- } \\
\text { Arts }\end{array}$ & $\begin{array}{c}\text { Droit } \\
\text { Économie } \\
\text { et Gestion }\end{array}$ & $\begin{array}{c}\text { Autres } \\
\text { DEUG } \\
\text { SHS }\end{array}$ \\
\hline Moyenne (en \%) & 7,3 & 22,5 & 12,6 & 22,9 & 18,1 & 9,7 & 18,3 \\
\hline Écart type & 8,2 & 16,3 & 15,5 & 24,1 & 15,8 & 13,9 & 143 \\
\hline
\end{tabular}

mettre en place des options lorsque le nombre d'étudiants susceptibles de les suivre est important.

Sur l'ensemble de l'échantillon, 29,1\% des filières offrent une orientation à l'issue du premier semestre. Cette situation est particulièrement fréquente en Sciences. Les antennes universitaires, en dépit de leurs faibles effectifs, proposent généralement les mêmes spécialisations que les universités "mères". La spécialisation peut dans certains cas reposer sur une professionnalisation de la filière. Cette professionnalisation (ou plus exactement pré-professionnalisation) existe également sous forme de modules préparant aux concours d'entrée dans la fonction publique ou dans les Instituts Universitaires de Formation des Maîtres (IUFM) ou bien encore sous forme de stages. Enfin, quelques rares filières créent des «sous-filières" d'excellence (à l'exemple des préparations aux concours d'entrée aux Grandes Écoles).

La diversification des filières se place sur un continuum où l'on trouve à une extrémité les filières qui privilégient l'uniformité des cursus (pas d'orientation, peu d'enseignements optionnels, pas de module spécifique) et, à l'opposé, des filières qui adoptent un système très diversifié, avec une spécialisation précoce et une pré-professionnalisation prononcée. La filière Droit (et dans une moindre mesure la filière AES) semble le mieux caractériser le premier type d'organisation alors que ce sont les filières scientifiques qui sont les plus représentatives du second. Entre ces extrémités se trouvent les filières de Sciences Humaines dans lesquelles les possibilités de choix optionnels sont importantes.

Le dernier élément qui différencie les filières au plan des curricula est l'importance accordée à chaque discipline en termes de volume horaire et de certification. Il est difficile à ce niveau de détail de construire des indicateurs pouvant rendre compte efficacement de la variété des situations. On se limitera à l'exemple du DEUG AES qui illustre sans doute le mieux cette variété. Créée en 1973, la filière AES est pluridisciplinaire. Les enseignements portent notamment sur le Droit, l'Économie, la Gestion et les Sciences Humaines et Sociales. Toutefois, les établissements sont libres de fixer le volume d'enseignement correspondant à chaque discipline. Lorsque l'on interroge les responsables de la filière sur la discipline dominante, certains avancent le Droit, d'autres I'Économie ou la Gestion mais également d'autres disciplines telles que l'Histoire ou les Statistiques. L'exemple de cette filière ne constitue pas un cas isolé : la différenciation des programmes est également très fréquente en Sciences de la Vie où, par le jeu des options, ils acquièrent un caractère fortement local.

\section{Les modes de certification}

Les modes de certification sont étudiés ici en référence aux conditions générales de validation (semestrielle, annuelle; mode de compensation), aux conditions de rattrapage (session, conditions de conservation des notes initiales et de repêchage des étudiants) et à la valeur attribuée à chaque enseignement (pondération des modules et du contrôle continu).

Parmi les 155 filières le mode dominant est celui de la certification annuelle avec capitalisation modulaire : l'étudiant doit valider la première année (ou le premier niveau) pour prétendre suivre les enseignements de la seconde année. Cette validation suppose généralement l'obtention de la moyenne aux examens. Toutefois, certains étudiants dont la note se situe quelques points en dessous peuvent être repêchés par le jury sous certaines conditions (obtention d'une note mini- 
male dans chaque module, chaque discipline, chaque examen du contrôle terminal, etc.). La compensation entre les modules n'est pas systématiquement appliquée puisque $9,7 \%$ des filières n'adoptent pas ce système. Lorsqu'il est mis en place, $16,3 \%$ des filières exigent une note minimale (généralement de $8 / 20$ ) pour que la compensation s'applique. Le second mode de certification est celui de la capitalisation modulaire avec compensation à l'issue des deux années de DEUG. Contrairement au modèle précédent, l'obtention du DEUG n'est prononcée que lorsque l'étudiant s'est présenté à l'ensemble des modules des deux années et que la moyenne de ceux-ci est au moins égale à 10 sur 20. Enfin, un dernier modèle a émergé récemment : c'est celui de la validation semestrielle des acquis. L'étudiant doit obtenir la moyenne à l'issue de chaque semestre (ou chaque période) pour valider celui-ci et cumuler les quatre semestres pour acquérir le DEUG.

Le contrôle des connaissances s'effectue le plus souvent lors de trois sessions d'examens: la session anticipée, la session terminale et la session de rattrapage. Si celle-ci se déroule généralement en septembre, certaines universités $(9,1 \%)$ organisent la session de rattrapage quelques jours ou quelques semaines après la session terminale. Les modalités de conservation des notes obtenues lors de la première session sont très variables. Les notes de contrôle continu sont généralement reportées tandis que les notes d'examens terminaux le sont sous certaines conditions. Dans $62,1 \%$ des cas, la note est reportée si elle est supérieure ou égale à 10 sur 20 alors que dans $8 \%$ des cas c'est la meilleure note des deux sessions qui est prise en considération. Enfin, $23 \%$ des filières ne prennent en compte que la note obtenue lors de la session de rattrapage.
Dans près d'un cas sur cinq $(18,9 \%)$, le poids du contrôle continu est proportionnel à sa part dans le volume des enseignements. En revanche $36,4 \%$ des filières donnent davantage de poids au contrôle continu et $44,7 \%$ adoptent la position opposée. Le poids du contrôle continu dans le contrôle des connaissances est en moyenne de $46,9 \%$ et varie de $35 \%$ en AES à $59,4 \%$ dans les disciplines littéraires. À nouveau la variété des situations inter-sites apparaît très importante si on en juge par la valeur de l'écart-type de cette proportion dans les différentes filières. Cette situation se retrouve dans la pondération respective des modules obligatoires et des modules optionnels. Si on prend l'exemple de la Psychologie à Dijon et Toulouse, on constate que dans la première université, le module optionnel a un coefficient 1 alors que les deux modules obligatoires ont chacun un coefficient 3 et qu'à Toulouse les deux modules obligatoires et les deux modules optionnels ont un poids équivalent.

\section{Les dispositifs d'aide à la réussite}

La dernière caractéristique distinctive de l'organisation pédagogique des DEUG observée dans cette recherche concerne les dispositifs particuliers mis en place pour lutter contre l'échec en première année. L'hétérogénéité croissante des publics a conduit certaines universités à développer des dispositifs destinés à faciliter l'adaptation, l'intégration et la réussite des étudiants. II s'agit du tutorat, notamment méthodologique, des séances ou des cycles de remise à niveau et, plus traditionnellement, de l'accueil des étudiants par les enseignants.

Mis en place dans certaines universités bien avant son institution officielle en 1996 (circulaire $n^{\circ}$ 96-246), le tutorat s'est développé là encore avec une intensité et des règles de fonctionnement particulières selon les sites. Le tutorat d'ac-

Tableau IV. - Part du contrôle continu dans le contrôle des connaissances

\begin{tabular}{|l|c|c|c|c|c|c|c|}
\hline \multicolumn{1}{|c|}{$\mathrm{N}=82$} & AES & Psychologie & $\begin{array}{c}\text { Sciences } \\
\text { de la Vie } \\
\text { Sciences } \\
\text { de la Terre }\end{array}$ & $\begin{array}{c}\text { Autres } \\
\text { DEUG } \\
\text { scientifiques }\end{array}$ & $\begin{array}{c}\text { Lettres } \\
\text { Langues- } \\
\text { Arts }\end{array}$ & $\begin{array}{c}\text { Droit } \\
\text { Économie } \\
\text { et Gestion }\end{array}$ & $\begin{array}{c}\text { Autres } \\
\text { DEUG } \\
\text { SHS }\end{array}$ \\
\hline Moyenne (en \%) & 35,0 & 37,3 & 36,8 & 35,7 & 59,4 & 42,3 & 50,9 \\
\hline Écart type & 9,7 & 17,5 & 8,5 & 15,0 & 30,4 & 9,1 & 19,0 \\
\hline
\end{tabular}


Tableau V. - Durée annuelle du tutorat selon les filières (en heures)

\begin{tabular}{|l|c|c|c|c|c|c|c|}
\hline \multicolumn{1}{|c|}{$\mathrm{N}=119$} & AES & Psychologie & $\begin{array}{c}\text { Sciences } \\
\text { de la Vie } \\
\text { Sciences } \\
\text { de la Terre }\end{array}$ & $\begin{array}{c}\text { Autres } \\
\text { DEUG } \\
\text { scientifiques }\end{array}$ & $\begin{array}{c}\text { Lettres } \\
\text { Langues- } \\
\text { Arts }\end{array}$ & $\begin{array}{c}\text { Droit } \\
\text { Économie } \\
\text { et Gestion }\end{array}$ & $\begin{array}{c}\text { Autres } \\
\text { DEUG } \\
\text { SHS }\end{array}$ \\
\hline Moyenne (en \%) & 27,2 & 17,6 & 47,1 & 13,5 & 34,3 & 37,8 & 25,0 \\
\hline Écart type & 31,1 & 14,0 & 38,3 & 15,9 & 48,5 & 32,4 & 22,9 \\
\hline
\end{tabular}

compagnement qui propose un suivi et une aide méthodologique aux étudiants est la forme la plus fréquente $(85,4 \%$ des filières). Près de trois filières sur quatre $(71,5 \%)$ offrent également un tutorat d'accueil. Mais il existe aussi d'autres formes de tutorat, telles que l'« initiation à l'informatique », le "tutorat documentaire », le suivi personnalisé des redoublants ou la préparation des examens de la seconde session. Le tutorat, notamment le tutorat d'accompagnement, est moins fréquent dans les antennes universitaires $(62,5 \%$ contre $88,1 \%$ dans les universitésmères) où l'encadrement pédagogique est considéré comme plus favorable.

Le volume horaire annuel du tutorat par étudiant bénéficiaire est relativement plus important en Sciences de la Vie (en moyenne 47,1 heures par an) qu'en AES $(27,2)$ ou qu'en Psychologie $(17,6$ heures). Mais, au-delà des écarts entre filières, ce sont davantage les disparités entre les universités qui resurgissent. Le nombre d'heures de tutorat varie par exemple en AES de $16 \mathrm{~h}$ à Lyon II à $120 \mathrm{~h}$ à l'université d'Artois.

En marge du tutorat, près d'une filière sur cinq $(19,9 \%)$ propose un dispositif de "remise à niveau ". L'examen précis de ces dispositifs révèle cependant que la frontière entre la remise à niveau et le tutorat est parfois difficile à établir. II peut s'agir en effet de quelques heures de "soutien » assurées par des enseignants, le plus souvent dès le début de l'année universitaire, mais aussi de cycles particuliers. Des dispositifs de soutien particuliers sont parfois organisés entre juin et septembre pour préparer la session de rattrapage. Des évaluations limitées à l'université de Bourgogne ont mis en évidence la faible efficacité de ces dispositifs (Michaut, 1997 ; M'piayi, 1999).

Les rencontres étudiants-enseignants constituent la forme la plus traditionnelle de prise en charge des étudiants en difficulté. Ces rencontres peuvent être informelles ou se situer dans le cadre de permanences assurées par les enseignants. La tenue systématique de permanences par l'ensemble des enseignants ne concerne que $16 \%$ des filières. À l'opposé, $31,9 \%$ des filières n'assurent aucune permanence.

Les permanences sont peu fréquentes dans les universités à faible effectif, en particulier dans les antennes universitaires (12\% contre $34,0 \%$ dans les universités mères). Globalement, les filières à tradition littéraire organisent plus souvent des permanences notamment en Psychologie. À l'opposé, les filières scientifiques privilégient les entrevues informelles.

À titre de synthèse, une analyse factorielle présente les liens existant entre les différentes variables associées à l'organisation des DEUG. L'axe principal, expliquant $18,1 \%$ de l'inertie totale, oppose les filières adoptant une certification modulaire, une "aide " importante aux étudiants (tutorat, mise à niveau et permanence des enseignants pour rencontrer les étudiants) caractérisées par un volume d'enseignement relativement faible, un poids important des options (plus de $10 \%$ du volume annuel d'enseignement) et du contrôle continu ( $40 \%$ de la certification finale) et les filières offrant un volume d'enseignement supérieur à la moyenne et une organisation des cours laissant peu de place aux options. Le deuxième axe oppose les filières procédant à une spécialisation dès la première année de DEUG à celles ne procédant pas à des différenciations de cursus. Cet axe oppose également les filières dont les TD/TP représentent plus de la moitié du volume global d'enseignement aux filières qui privilégient les cours magistraux.

L'introduction des filières en tant que variables supplémentaires révèle globalement trois profils: le premier regroupe les filières scientifiques où les 


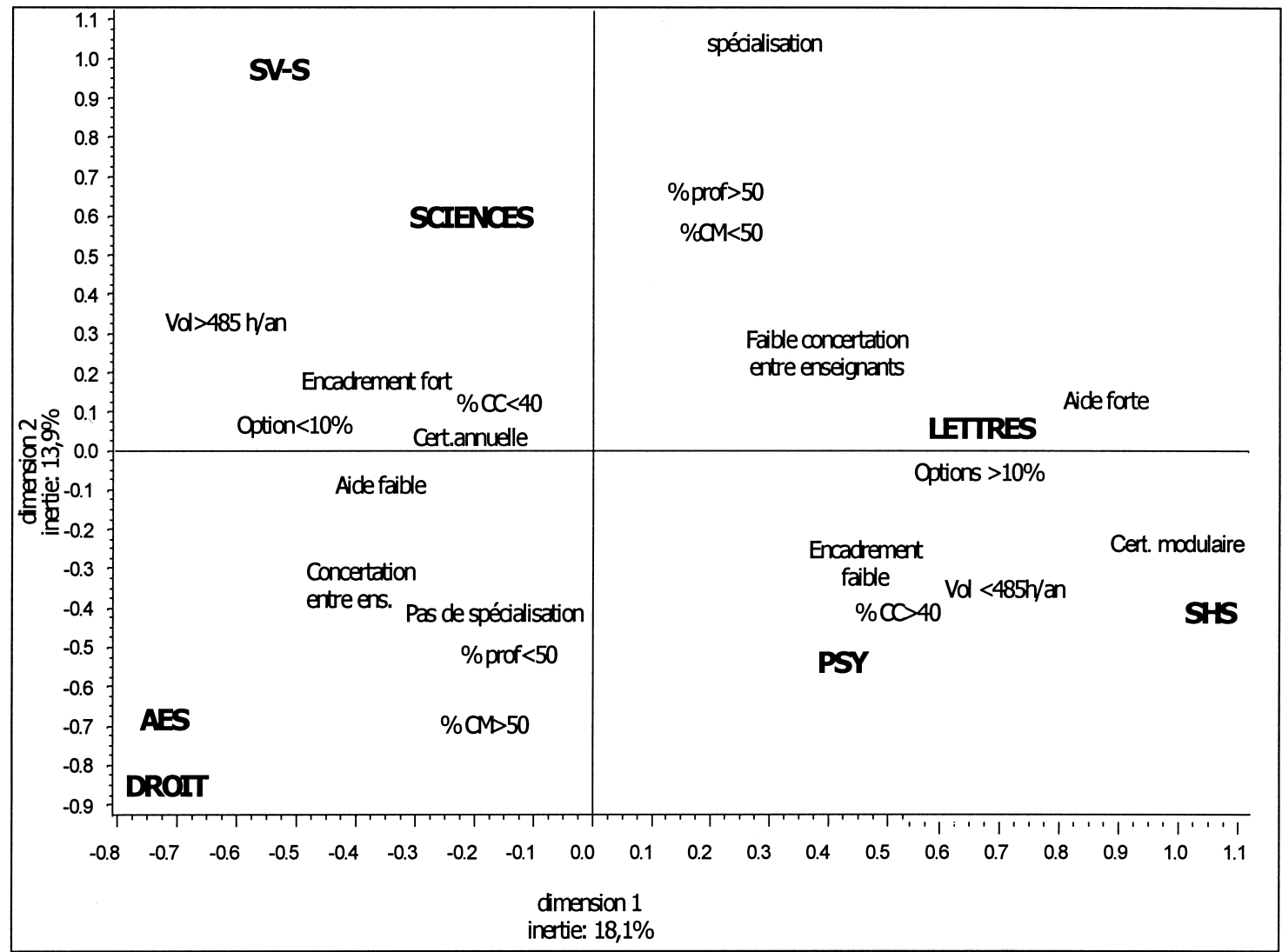

spécialisations, le volume d'enseignement et la représentation des enseignants statutaires sont importants. Un deuxième profil regroupe les filières Droit, Économie et AES dans lesquelles les spécialisations sont relativement rares et l'enseignement assuré plus souvent par les "nouveaux " enseignants du supérieur (PRAG, ATER, etc.). Enfin, le dernier groupe est composé des filières Littéraires au sens large (Lettres, Langues, Arts) et des filières des Sciences Humaines (Psychologie, Histoire et Géographie). Toutefois, ces filières ne se situent pas au centre de chaque groupe mais gravitent autour d'eux, attestant de la forte variété des situations d'enseignement entre les universités. En d'autres termes, chaque filière est proche d'une organisation particulière sans pour autant caractériser cette organisation.

Au terme de ce bilan, même partiel, des modes d'organisation des DEUG, il ressort avec évidence que les universités ont profité de l'autonomie de plus en plus grande qui leur a été accordée en matière d'organisation des enseignements. Ces modes d'organisation diffèrent de façon importante entre les filières mais surtout, à filière identique, selon les sites. De fait les étudiants inscrits dans une même filière sont soumis à des conditions d'enseignement souvent très différentes selon l'université fréquentée. Il devient alors intéressant d'étudier d'éventuelle relations entre la variété des modes d'organisation et les écarts en termes de réussite qui s'observent également d'une université à l'autre.

\section{INFLUENCE DES MODES D'ORGANISATION SUR LA RÉUSSITE}

Les taux de réussite calculés en référence aux inscrits apparaissent faibles quelle que soit la filière. En Psychologie et en AES, les taux observés 
Tableau VI. - Taux de réussite final ( $1^{\text {re }}$ et $2^{\text {nde }}$ session) selon les filières (en \%)

\begin{tabular}{|l|c|c|c|c|c|c|c|}
\hline \multicolumn{1}{|c|}{$\mathrm{N}=112$} & AES & Psychologie & $\begin{array}{c}\text { Sciences } \\
\text { de la Vie } \\
\text { Sciences } \\
\text { de la Terre }\end{array}$ & $\begin{array}{c}\text { Autres } \\
\text { DEUG } \\
\text { scientifiques }\end{array}$ & $\begin{array}{c}\text { Lettres } \\
\text { Langues- } \\
\text { Arts }\end{array}$ & $\begin{array}{c}\text { Droit } \\
\text { Économie } \\
\text { et Gestion }\end{array}$ & $\begin{array}{c}\text { Autres } \\
\text { DEUG } \\
\text { SHS }\end{array}$ \\
\hline Moyenne (en \%) & 39,8 & 38,2 & 47,5 & 50,9 & 52,3 & 47,3 & 47,5 \\
\hline Écart type & 7,1 & 12,9 & 12,8 & 10,4 & 20,4 & 12,1 & 15,4 \\
\hline
\end{tabular}
Tableau VII. - Part des étudiants absents à la première session par rapport aux inscrits
selon les filières (en \%)

\begin{tabular}{|l|c|c|c|c|c|c|c|}
\hline \multicolumn{1}{|c|}{$\mathrm{N}=112$} & AES & Psychologie & $\begin{array}{c}\text { Sciences } \\
\text { de la Vie } \\
\text { Sciences } \\
\text { de la Terre }\end{array}$ & $\begin{array}{c}\text { Autres } \\
\text { DEUG } \\
\text { scientifiques }\end{array}$ & $\begin{array}{c}\text { Lettres } \\
\text { Langues- } \\
\text { Arts }\end{array}$ & $\begin{array}{c}\text { Droit } \\
\text { Économie } \\
\text { et Gestion }\end{array}$ & $\begin{array}{c}\text { Autres } \\
\text { DEUG } \\
\text { SHS }\end{array}$ \\
\hline Moyenne (en \%) & 20,2 & 20,5 & 13,7 & 8,8 & 16,9 & 20,8 & 18,1 \\
\hline Écart type & 10,8 & 12,8 & 8,6 & 8,1 & 12,8 & 13,1 & 10,1 \\
\hline
\end{tabular}

sont en moyenne inférieurs à $40 \%$. Ils dépassent à peine $52 \%$ dans les filières les moins sélectives (Lettres). Si ces informations sont connues, ce qui est sans doute nouveau, c'est qu'à l'image des modes d'organisation, les taux de réussite varient de façon très importante selon les sites pour de mêmes filières. En témoignent les mesures de dispersion présentées dans le tableau 6.

La référence aux inscrits, qui prend en compte des abandons très précoces, associés à des doubles inscriptions ou plus souvent à des inscriptions de "précaution" (préparation de concours, attentes des résultats d'une candidature dans le secteur sélectif,...), sous-estime les taux de réussite réels. Le calcul des taux de réussite en référence aux seuls présents aux examens ne correspond pas pour autant à ces taux de réussite réels dans la mesure où se trouvent exclus du calcul des étudiants qui ont abandonné après avoir anticipé un échec. Rapporté aux étudiants présents, le taux de réussite varie de $47,8 \%$ en Psychologie à $63,2 \%$ en Lettres et Langues.

La part des étudiants qui ne se présentent pas aux examens est plus importante dans les filières à dominante littéraire que dans les filières à domi- nante scientifique. Les indicateurs de dispersion sont à nouveau très élevés.

L'examen des relations entre modes d'organisation et réussite est basé sur l'estimation de modèles de régression linéaire. La différenciation des modes d'organisation selon les filières nous impose de tenir compte de cette dimension et donc de raisonner à "filière donnée ". Ceci sera fait au niveau de l'échantillon global en introduisant des variables muettes permettant de contrôler l'appartenance spécifique à une discipline et par l'estimation de modèles intra-filières pour celles qui sont les plus représentées dans l'échantillon (AES et Sciences de la Vie).

Les modèles présentés dans le tableau 8 examinent l'influence des principaux modes d'organisation sur la réussite (cumul des deux sessions) calculée par rapport aux inscrits. Le premier modèle se limite aux variables muettes associées aux différentes filières. En moyenne, et en référence aux filières de sciences humaines (Histoire et Géographie), seule la filière AES se distingue par un taux de réussite significativement plus faible.

Le second modèle porte sur les modes d'organisation généralement présentés comme les plus différenciateurs (volume annuel d'enseignement, 
Tableau VIII. - Déterminants du taux de réussite final (ensemble des deux sessions)

\begin{tabular}{|c|c|c|c|c|c|c|c|}
\hline \multirow{2}{*}{ Variable de référence } & \multirow{2}{*}{ Variable active } & \multicolumn{2}{|c|}{$\begin{array}{l}\text { Modèle } 1 \\
(\mathrm{n}=107)\end{array}$} & \multicolumn{2}{|c|}{$\begin{array}{c}\text { Modèle } 2 \\
(\mathrm{n}=107)\end{array}$} & \multicolumn{2}{|c|}{$\begin{array}{l}\text { Modèle } 3 \\
(\mathrm{n}=107)\end{array}$} \\
\hline & & Coeff. & Signif. & Coeff. & Signif. & Coeff. & Signif. \\
\hline \multirow{6}{*}{$\begin{array}{l}\text { Filière Sciences } \\
\text { Humaines }\end{array}$} & AES & $-10,06$ & ** & $-17,73$ & *** & $-17,0$ & *** \\
\hline & Psychologie & $-5,49$ & ns & $-3,61$ & ns & $-3,48$ & ns \\
\hline & Sciences de la Vie & $-3,10$ & ns & $-17,04$ & ** & $-16,65$ & ** \\
\hline & $\begin{array}{l}\text { Autres filières } \\
\text { scientifiques }\end{array}$ & 1,75 & ns & $-11,97$ & * & $-12,22$ & * \\
\hline & Lettres/Langues & 5,68 & ns & 1,42 & ns & 0,59 & ns \\
\hline & Droit/Économie & $-2,41$ & ns & $-12,10$ & * & $-11,04$ & * \\
\hline \multicolumn{2}{|c|}{ Durée annuelle d'enseignement } & & & 0,070 & * & 0,071 & * \\
\hline \multicolumn{2}{|c|}{ Proportion de cours magistraux } & & & 0,008 & ns & 0,018 & ns \\
\hline \multicolumn{2}{|c|}{$\begin{array}{l}\text { Taux d'encadrement } \\
\text { (nb. d'étudiants/nb. d'enseignants) }\end{array}$} & & & $-0,17$ & ns & $-0,14$ & ns \\
\hline \multicolumn{2}{|c|}{ Proportion de Professeurs des universités } & & & 0,092 & ns & $-0,056$ & ns \\
\hline $\begin{array}{l}\text { Pas de concertation } \\
\text { entre enseignant }\end{array}$ & $\begin{array}{l}\text { Concertation } \\
\text { entre enseignants }\end{array}$ & & & & & $-1,86$ & ns \\
\hline Pas de spécialisation & Spécialisation & & & & & 3,54 & ns \\
\hline \multirow{2}{*}{$\begin{array}{l}\text { Pas de tutorat } \\
\text { et de permanence } \\
\text { des enseignants }\end{array}$} & $\begin{array}{l}\text { Tutorat et permanence } \\
\text { des enseignants }\end{array}$ & & & & & 1,12 & ns \\
\hline & Constante & 49,84 & $\star * *$ & 23,70 & * & 21,77 & ns \\
\hline \multicolumn{2}{|c|}{$\%$ de variance expliquée $\mathrm{R}^{2}$ ajusté } & \multicolumn{2}{|c|}{$12,1 \%$} & \multicolumn{2}{|c|}{$17,9 \%$} & \multicolumn{2}{|c|}{$19,5 \%$} \\
\hline
\end{tabular}

Significativité du $\mathrm{t}$ de Student : ${ }^{*}$ : significatif au seuil de 0,$10 ;{ }^{* *}: 0,05 ;{ }^{* \star *}: 0,01$.

proportion de cours magistraux, taux d'encadrement enseignant et proportion de Professeurs des Universités). Le volume horaire annuel exerce, à filière donnée, un impact positif et significatif sur la réussite. À durée annuelle d'enseignement comparable, on observe à présent des écarts significatifs de réussite entre filières. Ceci revient à constater par exemple, que les Sciences de la Vie, qui sont en moyenne d'une sélectivité comparable aux sciences humaines, obtiennent ce résultat malgré un volume d'enseignement plus important. Les trois autres variables considérées, en dépit de leur variation, n'entretiennent aucune relation statistique significative avec la réussite. L'introduction des variables mesurant la dimension plus qualitative de l'organisation (concertation entre enseignants pour la préparation des cours, spécialisation des enseignements, et aide aux étudiants) conduit au même résultat.

Le tableau 9 présente les estimations réalisées pour les deux filières les plus représentées dans notre échantillon (AES d'une part, Sciences de la Vie - Sciences de la Terre d'autre part). Les résultats obtenus sont tout aussi décevants. Ils manifestent à nouveau une quasi-absence de relation entre les indicateurs des principaux modes d'organisation et la réussite. Compte tenu de la faiblesse du nombre d'observations, nous avons testé la robustesse des résultats en analysant pour chaque filière l'effet des différentes variables considérées une à une. Tous les résultats présentés dans le tableau 8 ont été confirmés en termes de sens (positif/négatif) et de significativité. 
Tableau IX. - Influence de l'organisation pédagogique sur le taux de réussite final (AES et SV-ST)

\begin{tabular}{|c|c|c|c|c|c|}
\hline \multirow[t]{2}{*}{ Variable de référence } & \multirow[t]{2}{*}{ Variable active } & \multicolumn{2}{|c|}{$\begin{array}{c}\text { Modèle } 1 \\
\text { AES } \\
(n=21)\end{array}$} & \multicolumn{2}{|c|}{$\begin{array}{c}\text { Modèle } 2 \\
\text { SV-ST } \\
(\mathrm{n}=21)\end{array}$} \\
\hline & & Coeff. & Signif. & Coeff. & Signif \\
\hline \multicolumn{2}{|l|}{ Durée annuelle d'enseignement } & 0,091 & ** & $-0,027$ & ns \\
\hline \multicolumn{2}{|l|}{ Proportion de cours magistraux } & 0,14 & ns & $-0,77$ & * \\
\hline \multicolumn{2}{|c|}{ Taux d'encadrement (nb. d'étudiants/nb. d'enseignants) } & 0,16 & ns & $-1,96$ & ns \\
\hline \multicolumn{2}{|c|}{ Proportion de Professeurs des universités } & 0,14 & ns & 1,16 & *** \\
\hline $\begin{array}{l}\text { Pas de concertation } \\
\text { entre enseignant }\end{array}$ & Concertation entre enseignants & $-7,94$ & ** & 2,14 & ns \\
\hline Pas de spécialisation & Spécialisation & 2,90 & ns & $-1,30$ & ns \\
\hline \multirow[t]{2}{*}{$\begin{array}{l}\text { Pas de tutorat et de permanence } \\
\text { des enseignants }\end{array}$} & $\begin{array}{l}\text { Tutorat et permanence } \\
\text { des enseignants }\end{array}$ & 3,35 & ns & 9,69 & ns \\
\hline & Constante & 4,85 & ns & 21,77 & ns \\
\hline \multicolumn{2}{|l|}{$\%$ de variance expliquée $R^{2}$ ajusté } & \multicolumn{2}{|c|}{$25,3 \%$} & \multicolumn{2}{|c|}{$32,8 \%$} \\
\hline
\end{tabular}

L'effet positif du volume d'enseignement ne se manifeste significativement que dans la filière AES pour laquelle on observe, en outre, une influence négative de la concertation entre les enseignants. Ce résultat, a priori surprenant, pourrait se comprendre si la concertation entre enseignants avait pour conséquence un renforcement du niveau d'exigence. II serait éventuellement possible d'interpréter dans le même esprit les résultats constatés en Sciences de la Vie qui font apparaître une influence négative sur la réussite de la proportion de professeurs de rang $A$ dans le corps enseignant. En revanche, l'impact négatif de la proportion de cours magistraux pourrait être plus spécifique à cette filière pour laquelle l'importance des enseignements dirigés et pratiques est associée à la réussite.

L'absence de relation entre modes d'organisation et réussite est à bien des égards surprenante. Ce résultat justifierait à lui seul la poursuite et l'extension de ce type d'investigation. II peut éventuellement tenir à la qualité des données collectées, et notamment à l'hétérogénéité des filières. La portée de cet argument est cependant limitée dans la mesure où des résultats comparables s'observent également lorsqu'on estime les modèles à l'intérieur de groupes homogènes au plan disciplinaire. Par ailleurs, et tout en reconnaissant que des informations plus précises pour- raient être obtenues sur les modes de fonctionnement, il est peu contestable que celles qui ont été considérées concernent des éléments importants de l'organisation pédagogique des filières, touchant à la fois les dotations et l'organisation institutionnelle.

En s'interrogeant sur l'origine de cette quasiabsence de relations entre les modes d'organisation et la réussite, on peut évoquer deux explications possibles:

- la première renvoie à la non prise en compte dans les modèles précédents (faute de données adéquates) des caractéristiques du public étudiant propre à chaque site. II faudrait cependant pour admettre cette explication considérer que les modes d'organisation choisis par les différents sites répondent précisément aux difficultés associées au public accueilli (l'efficacité des modes d'organisation en matière de réussite seraient alors exactement "compensée " par un effet négatif lié aux caractéristiques des étudiants) ;

- la seconde explication renvoie au caractère " contextualisé » de la mesure de la réussite. Les travaux réalisés dans les niveaux d'enseignement pré-universitaire ont clairement mis en évidence le caractère local (en référence à une évaluation externe du niveau scolaire des élèves) de la notation et des décisions d'orientation. Les résultats observés pourraient renvoyer au même phéno- 
mène, les filières universitaires ne disposant pas d'une référence nationale en ce domaine, développeraient des pratiques spécifiques en matière de notation et d'orientation, pratiques dépendant partiellement des caractéristiques des étudiants accueillis.

Une situation de ce genre est sans doute inévitable compte tenu de la relative souplesse des programmes nationaux et de l'autonomie dont jouissent les établissements. Elle pourrait conduire à s'interroger sur le caractère national des diplômes délivrés. Elle pose en outre un problème méthodologique important. Si un résultat de ce type devait se confirmer, cela mettrait, en effet, directement en cause la possibilité de mesurer de manière fiable l'efficacité des modes d'organisation (l'efficacité des dotations) sur la base d'indicateurs de réussite dont le niveau serait endogène au fonctionnement de l'institution. Le défaut d'épreuves communes et l'absence de véritables examens nationaux constitueraient ainsi un obstacle majeur à l'évaluation du fonctionnement pédagogique des établissements universitaires.

Jean-Pierre Jarousse Christophe Michaut

Université de Bourgogne, IREDU-CNRS

\section{BIBLIOGRAPHIE}

DUBOIS P. (1997). - L'organisation des universités: complexification, diversification, rationalisation, évaluation. Sociétés Contemporaines, $n^{\circ} 28$, p. 13-32.

DURU-BELLAT M., JAROUSSE J-P., LEROY C., MICHAUT C. et al. (1999). - Hétérogénéité et réussite en premier cycle universitaire. Profils individuels et fonctionnement pédagogique. IREDUCNRS-Université de Bourgogne. Rapport pour le CNCRE, $181 \mathrm{p}$.

FELOUZIS G. (dir.), ABADIE F., ANDRIEU J. (1998). Les nouvelles inégalités à l'université : parcours étudiants et organisation des études dans trois disciplines universitaires. LAPSAC-Université de Bordeaux 2.

M'PIAYI A. (1999). - Les déterminants de la réussite universitaire : le cas du DEUG B à l'Université de Bourgogne. Thèse de Doctorat, Université de Bourgogne-IREDU, $392 \mathrm{p}$.

MICHAUT C. (1997). - La réussite et l'orientation à I'Université. Le cas du DEUG Sciences de la Vie. Mémoire de DEA, Université de Bourgogne-IREDU, $83 \mathrm{p}$.

MICHAUT C. (2000). - L'influence du contexte universitaire sur la réussite des étudiants. Thèse de Doctorat, Université de Bourgogne-IREDU. 
